\title{
THE
}

2005

\section{A Multi-Index GEM Technique and Its Application to the Southwestern Japan/East Sea}

\author{
Jae-Hun Park \\ University of Rhode Island \\ D. Randolph Watts \\ University of Rhode Island \\ Karen L. Tracey \\ University of Rhode Island \\ Douglas A. Mitchell
}

Follow this and additional works at: https://digitalcommons.uri.edu/gsofacpubs

Terms of Use

All rights reserved under copyright.

\section{Citation/Publisher Attribution}

Park, J., Watts, D. R., Tracey, K. L., \& Mitchell, D. (2005). A Multi-Index GEM Technique and Its Application to the Southwestern Japan/East Sea. Journal of Atmospheric and Oceanic Technology, 22, 1282-1293. doi: 10.1175/JTECH1778.1

Available at: https://doi.org/10.1175/JTECH1778.1

This Article is brought to you for free and open access by the Graduate School of Oceanography at DigitalCommons@URI. It has been accepted for inclusion in Graduate School of Oceanography Faculty Publications by an authorized administrator of DigitalCommons@URI. For more information, please contact digitalcommons-group@uri.edu. 


\title{
A Multi-Index GEM Technique and Its Application to the Southwestern Japan/East Sea
}

\author{
Jae-Hun Park, D. Randolph Watts, and Karen L. Tracey \\ Graduate School of Oceanography, University of Rhode Island, Narragansett, Rhode Island \\ Douglas A. Mitchell \\ Naval Research Laboratory, Stennis Space Center, Mississippi
}

(Manuscript received 22 April 2004, in final form 14 November 2004)

\begin{abstract}
This paper demonstrates a new gravest empirical mode (GEM) technique that constructs multi-index lookup tables of temperature $(T)$ and specific volume anomalies $(\delta)$ using historical hydrocasts as a function of three indices: round-trip travel time $(\tau)$ from sea floor to the surface, sea surface temperature, and pressure. Moreover, the historical hydrocasts are separated into non-mixed-layer (NML) and mixed-layer (ML) groups, and a single GEM field is constructed for each group. This is called the MI-GEM technique. The appropriate dates for MI-GEM fields are determined by the monthly distribution of the number of NML and ML profiles in the historical hydrocasts, which are also well correlated with the strength of the winds during the $2 \mathrm{yr}$ of observations. The $T$ and $\delta$ profiles that are determined by this MI-GEM technique capture $92 \%$ and $88 \%$ of the $T$ and $\delta$ variances in the depth range of $0-200 \mathrm{db}$. These values reduce by about one-third of the unexplained error variance of the residual GEM, which was recently developed and applied to the optimal interpolated $\tau$ data in the southwestern Japan/East Sea (JES) by Mitchell et al. Comparisons with the in situ CTD casts demonstrate that the MI-GEM technique almost always produces improved full water column profiles of $T$ and $\delta$. Whereas the residual GEM estimates had exhibited qualitatively erroneous features like $T$ inversions in the near-surface layer and too thin or thick intermediate water layers in some regions, the MI-GEM estimates avoid those problems, which were inherent to the residual GEM technique in the southwestern JES.
\end{abstract}

\section{Introduction}

The gravest empirical mode (GEM) technique is a method that is used to estimate vertical profiles of temperature $(T)$, salinity $(S)$, and specific volume anomaly $(\delta)$ from a vertically integrated proxy, such as roundtrip acoustic travel time $(\tau)$ from the bottom to the surface, geopotential height, or heat content (Meinen and Watts 2000; Watts et al. 2001). The standard GEM technique produces two-index lookup tables (GEM fields) of $T, S$, and $\delta$ from historical hydrocast data as a function of $\tau$ (or another index or proxy) and pressure. This standard GEM technique has been applied successfully to the North Atlantic (Meinen and Watts

Corresponding author address: Jae-Hun Park, Graduate School of Oceanography, University of Rhode Island, 215 South Ferry Road, Narragansett, RI 02882-1197.

E-mail: jpark@gso.uri.edu
2000) and Antarctic Circumpolar (Sun and Watts 2001) Currents. An improved standard GEM technique, which removes the seasonal signals in the upper layer prior to the GEM field computation to reduce the associated errors, has been developed and applied to the subantarctic front (Watts et al. 2001) and the Kuroshio south of Japan (Book et al. 2002). However, Mitchell et al. (2004) recently demonstrated that the southwestern Japan/East Sea (JES), called the Ulleung Basin (UB), is not suitable to this improved standard GEM technique because variations in the shallow permanent thermocline occur at the same depths as the seasonal signal, which varies in vertical structure, amplitude, and phase from one location to another, even within this limited region. Therefore, they modified the method to estimate anomalies relative to a climatology, referred to as the residual GEM technique, as follows.

The residual GEM technique builds upon a climatology, such as the U.S. Navy's Modular Ocean Data As- 
similation System (MODAS). Mitchell et al. (2005) used this to represent the spatiotemporal annual signal, and used historical hydrocasts to estimate the residual (i.e., anomaly) $T$ and $\delta$ variability from those, indexed by residual acoustic travel time as a proxy variable. The residual GEM fields were additionally grouped under three classes of residual sea surface temperature (SST), which significantly reduced the errors near the surface. Mitchell et al. (2005) produced accurate daily $T$ maps at a $100-\mathrm{m}$ depth in the UB for $2 \mathrm{yr}$, using the residual GEM technique to interpret $\tau$ data from pressure sensor-equipped inverted echo sounders (PIESs).

In the UB, dynamics of the intermediate water is of great interest. In particular, a water mass with a vertical $S$ minimum that is accompanied by a dissolved oxygen maximum may be found in a depth range of 100-400 m. This water mass has the temperature and potential density range from $5^{\circ}$ to $1^{\circ} \mathrm{C}$ and from 26.9 to $27.3 \sigma_{\theta}$, and is hypothesized to originate from subducted water along the northern region of the subpolar front. Kim and Chung (1984) found it in the UB and named it the East Sea Intermediate Water (ESIW). Although there are several observational studies showing the distribution and circulation of the ESIW in the UB (e.g., Kim et al. 1991; Shin et al. 1998), those hydrographic studies produced intermittent snapshots of the ESIW circulation that were insufficient to understand its source and circulation. In contrast to the above studies, the dynamic height estimates from our PIES array can provide daily maps of the circulation at intermediate depths. However, some interpretations that used the residual GEM estimates were found to be erroneous regarding intermediate water temperature structures, by estimating an unrealistically thin or thick ESIW layer. Comparisons of the residual GEM results against concurrent in situ hydrocasts revealed that the residual GEM sometimes produced erroneous $T$ and $\delta$ inversions near the surface layer. In addition, the base of the thermocline (pycnocline) at $1^{\circ} \mathrm{C}\left(27.3 \sigma_{\theta}\right)$ was qualitatively too shallow or too deep in some regions. Although the residual GEM had improved upon the previous GEM estimates of the thermal structure in the $\mathrm{UB}$, and, indeed, worked quite well for mapping the temperature at a 100-m depth, it unavoidably produced the inversions and other errors in near-surface waters and was complicated to apply.

The problems with the residual GEM estimates motivated us to develop a further-improved GEM technique that is applicable to the UB. In this paper, we demonstrate a multi-index GEM technique, which uses satellite-mapped SST as an additional parameter to make full multi-index lookup tables for $T$ and $\delta$ versus pressure, and also distinguishes between non-mixed- layer (NML) and mixed-layer (ML) profiles, as described later. Herein we call this the MI-GEM technique. We apply this new GEM technique to the $\tau$ measurements from an array of PIES in the UB.

\section{Data and methods}

We use $\tau$ records measured by an array of PIES in the UB, a set of 2248 historical hydrocasts, 441 in situ CTD casts from the Korea Ocean Research and Development Institute (KORDI) and the Korea Oceanographic Data Center (KODC), and SST from the Tropical Rainfall Measuring Mission (TRMM) satellite observations. These data are the same as those used in Mitchell et al. (2004). In addition, time series of Navy Operational Global Atmospheric Prediction System (NOGAPS) wind stress, on a degree grid with a time interval of $12 \mathrm{~h}$, were used to distinguish time intervals when a deepened ML should occur. A brief description of $\tau$ and SST datasets is presented here. For more details, see Mitchell et al. (2004).

An array of 25 PIESs, designed to have 55-60-km spacing covering the UB, was deployed during 19992001 (Fig. 1). The PIES measured $\tau$ each hour with a 0.05-ms precision. Two PIESs at P32 and P41 were lost, probably because of deep-crab-fishing activity. Fiveday low-pass filtering was applied to the $\tau$ records to focus on geostrophic signals. Then, the $\tau$ records were subsampled at 1200 UTC every day for 736 days from 16 June 1999 to 20 June 2001.

Application of optimal interpolation (OI) mapping to the subsampled $\tau$ requires the selection of a standardized reference level, because each PIES measured $\tau$ at its individual bottom depth. Mitchell et al. (2004) chose a reference level of $500 \mathrm{db}$ and calculated a linear relationship between $\tau$ and $\tau_{500}$ at each station from deep hydrocasts extending to the bottom. The round trip $\tau$ between $0 \mathrm{db}$ and $p$ is

$$
\tau_{p}=2 \int_{0}^{p} \frac{d p^{\prime}}{\rho g_{0} c},
$$

where $\rho\left(p^{\prime}\right)$ is density, $g_{0}$ the constant acceleration of gravity at $9.8 \mathrm{~m} \mathrm{~s}^{-2}$, and $c\left(p^{\prime}\right)$ the sound speed (see Meinen and Watts 1997). The 500-db level was selected as the index level in order to utilize the relatively large number of hydrocasts that only extended to this depth. Because the waters in the JES below $500 \mathrm{db}$ are nearly homogeneous, a simple linear relationship together with the CTDs shifts the measured $\tau_{p}$ records that are collected at various depths $p$ to a calibrated $\tau_{500}$ [similar to the methods employed by Tracey et al. (1997) and Meinen and Watts (1998)]. The $\tau_{500}$ accu- 


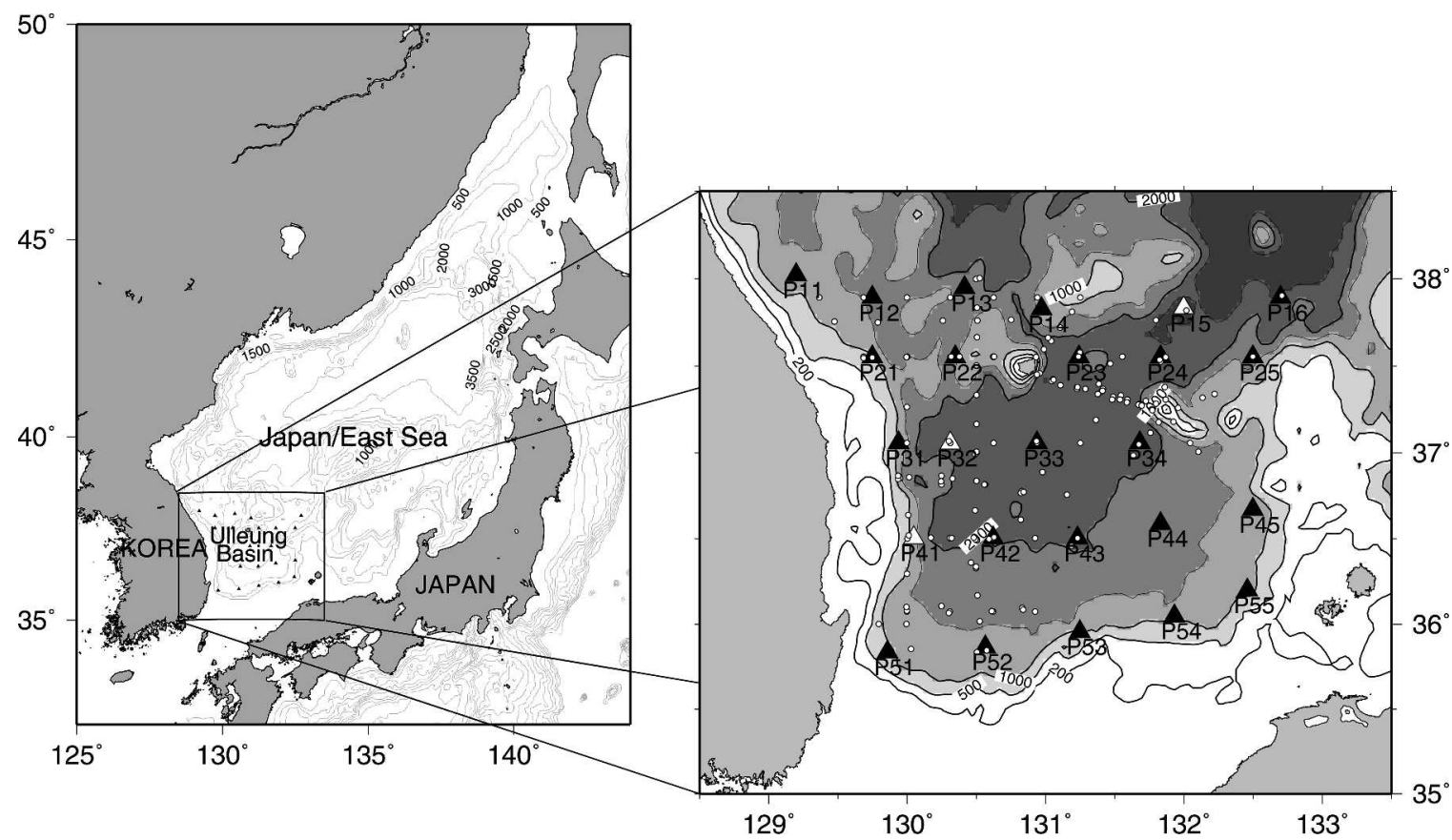

FIG. 1. The Japan/East Sea. Solid triangles indicate PIES sites and white circles are in situ CTD surveys by KORDI and KODC. Bathymetry contours are in meters.

racy is about $1.1 \mathrm{~ms}$ when compared against several CTDs. Finally, the $\tau_{500}$ records were OI mapped on a $0.125^{\circ} \times 0.125^{\circ}$ grid in the UB using a $42-\mathrm{km}$ correlation length scale, as was used by Mitchell et al. (2004).

The TRMM Microwave Imager (TMI) radiometer with a $10.7-\mathrm{GHz}$ channel collects SST data through nonprecipitating clouds, which is a distinct advantage over the traditional infrared SST observations that require a cloud-free field of view. The TRMM SST data were downloaded from the Remote Sensing Systems Web site as daily 3-day averages and weekly composites on a $0.25^{\circ} \times 0.25^{\circ}$ grid covering the UB. After filling in missing values of SST by interpolation, we produced 736 daily time series of OI-mapped SST on the same grid as OI-mapped $\tau_{500}$ using the MODAS climatological SST as the mean field in the OI calculation.

\section{MI-GEM technique}

\section{a. Development of MI-GEM fields}

Mitchell et al. (2004) illustrated clearly the difficulties in constructing a GEM field in the UB. They showed two pairs of temperature profiles, for example (Fig. 2). One pair was collected at a single location in the summer- and wintertime, and the other pair was collected at different locations in the same month. Each profile pair has essentially the same $\tau_{500}$, but the ele- ments have different vertical shapes, for example, a warm surface layer with a shallow thermocline versus a mixed layer with a depressed thermocline. The standard GEM technique estimates a single $T$ profile from $\tau_{500}$ and, hence, the standard GEM fields cannot distinguish between these profile pairs. We utilize available SST data to improve the estimates. Mitchell et al. (2004) also used the SST data in their residual GEM technique, but they classified the SST into only three broad bins. In contrast, we selected SST bins having $1^{\circ} \mathrm{C}$ intervals from $5^{\circ}$ to $30^{\circ} \mathrm{C}$ in order to index with a well-resolved SST. In addition, our technique estimates the ML structure as described below. As Mitchell et al. (2004) mentioned, the incorrect estimation of the ML was the largest source of error in the residual GEM. Altogether, our MI-GEM technique depends on the presence or absence of an ML, and utilizes multi-index lookup tables of $T$ and $\delta$ as functions of $\tau_{500}$, SST, and $p$.

Figure 3 illustrates the difference between the standard GEM and MI-GEM techniques, using scatterplots of $T$ versus $\tau_{500}$. The standard two-index GEM technique (e.g., Watts et al. 2001) seeks a smooth, singlevalued relationship between the data at each $p$ and $\tau_{500}$, exemplified by the black curve on each scatterplot at 100, 200, and $300 \mathrm{db}$. The superimposed black curves are smoothing cubic-spline curves that are fitted to all of the $T$ data from the historical hydrocasts, as repre- 

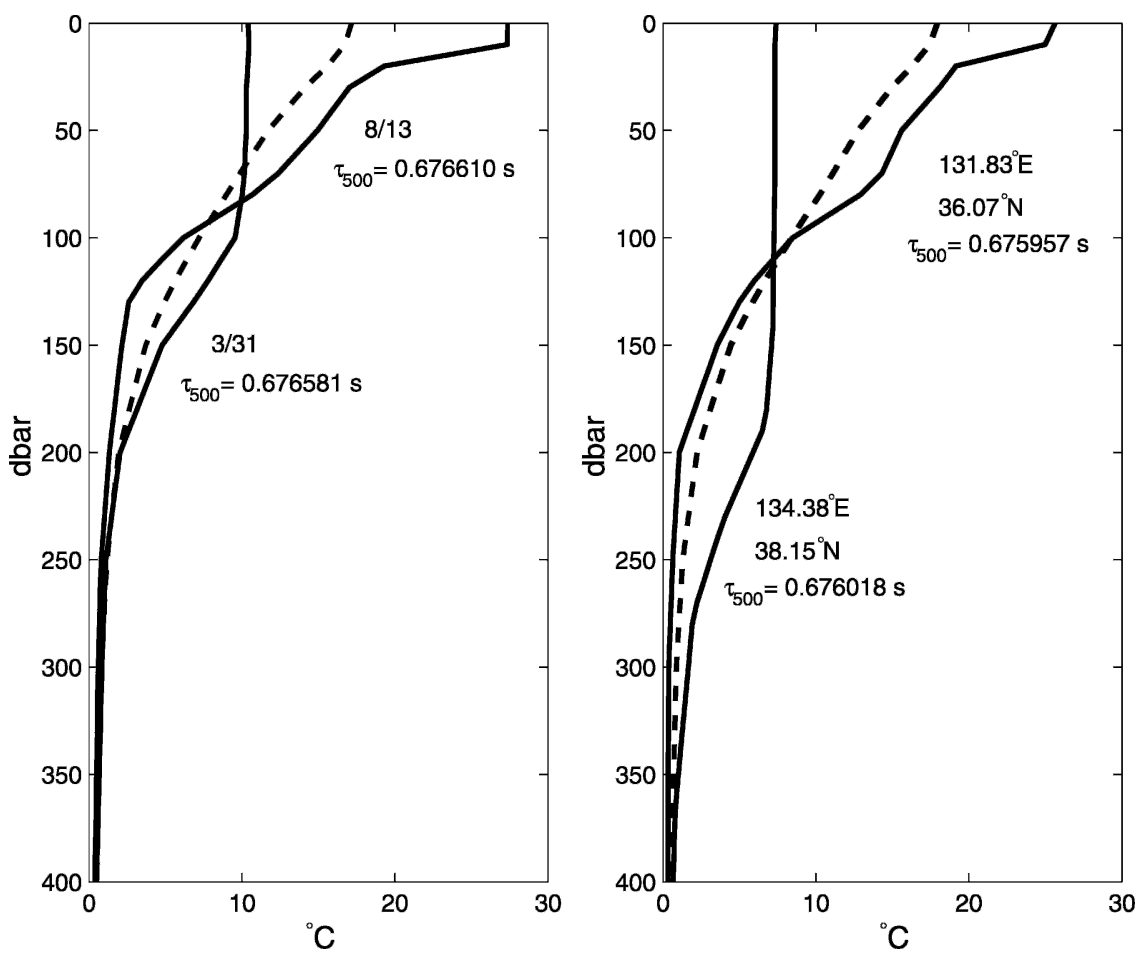

FIG. 2. (left) Hydrographic temperature profiles near $36.36^{\circ} \mathrm{N}, 134.08^{\circ} \mathrm{E}$ for Mar and Aug, illustrating the seasonally changing vertical structure. (right) Hydrographic temperature profiles for two locations that are $325 \mathrm{~km}$ apart during the month of Feb. In each panel, two measured temperature profiles (solid lines) with similar $\tau_{500}$ values are compared with profiles estimated from the standard GEM technique (dashed line) (reproduced from Mitchell et al. 2004).

sented by black dots. The standard 2D GEM lookup field of $T$, as a function of $\tau_{500}$ and $p$, is produced by fitting a cubic-spline curve to the $T$ versus $\tau_{500}$ data on each $10-\mathrm{db}$ pressure level between 0 and $500 \mathrm{db}$, generating 51 spline curves.

The MI-GEM technique regroups the same $T$ profiles into subsets that depend on SST and the presence or absence of a mixed layer. As an illustration for just one of the SST bins $\left(15.5^{\circ}-16.5^{\circ} \mathrm{C}\right)$, the red and blue curves in Fig. 3 are fitted to the subsets of NML and ML groups. When the temperature difference between 0 and $30 \mathrm{db}$ is larger (smaller) than $1{ }^{\circ} \mathrm{C}$, the profile was classified as being an NML (ML) group. At the 100-db level, the ML group has higher $T$ than that of the NML group when $\tau_{500}$ is less than $0.676 \mathrm{~s}$. The reverse is the case for the 200-db level. As $\tau_{500}$ decreases, the absolute differences of $T$ between the NML and ML groups increase at 100 and $200 \mathrm{db}$. At the 300-db level, the datafitted curves for both of the groups are consistent everywhere with the black curve. This relationship implies that a warm (cold) temperature in the UB at one layer is compensated by a cold (warm) temperature at the other layer to retain a selected $\tau_{500}$ value. The re- sults of this regrouping to utilize SST and ML information are shown in Fig. 4. The profiles were first grouped into NML and ML categories and sorted according to SST. Then, within $1{ }^{\circ} \mathrm{C}$ intervals of SST, cubic-spline curves were fitted on each pressure level at 10-db intervals to generate a standard GEM field of $T$ for each separate group as a function of $\tau_{500}$ and $p$. Last, it was necessary to fit smoothing cubic splines along the SST axis at each pressure level to make smoothly varying MI-GEM fields (Fig. 4). The same procedures were applied to construct MI-GEM fields of $\delta$.

To interpret PIES data using this new technique, we further need a criterion for switching between the NML and ML MI-GEM fields. The histogram in Fig. 5 exhibits the monthly distribution of the number of NML and ML profiles from the 2248 hydrocasts that are used to construct the NML and ML MI-GEMs. This histogram illustrates that NML and ML profiles predominantly occur during the summer and winter months, respectively. The 2-yr time series of NOGAPS basinaveraged wind stress through the UB (Fig. 5a) illustrates the significant seasonal variation, that is, strong (weak) wind during the winter (summer)-time, which 
(a) 100 dbar

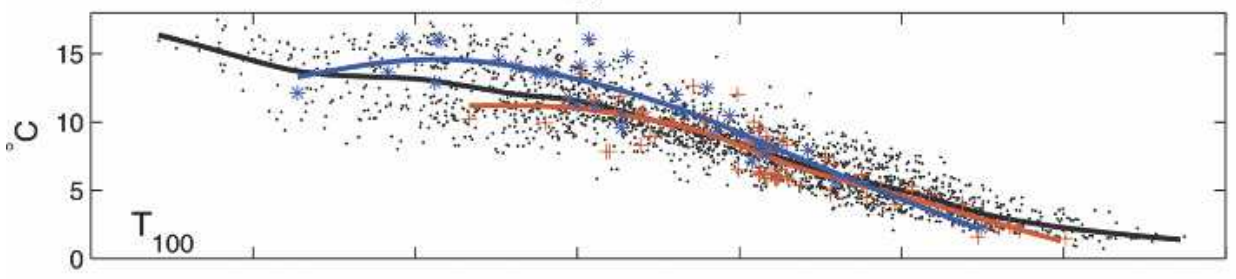

(b) 200 dbar

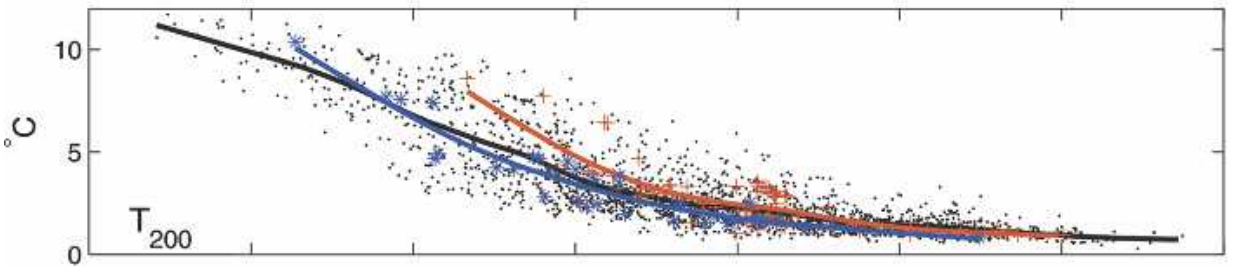

(c) $300 \mathrm{dbar}$

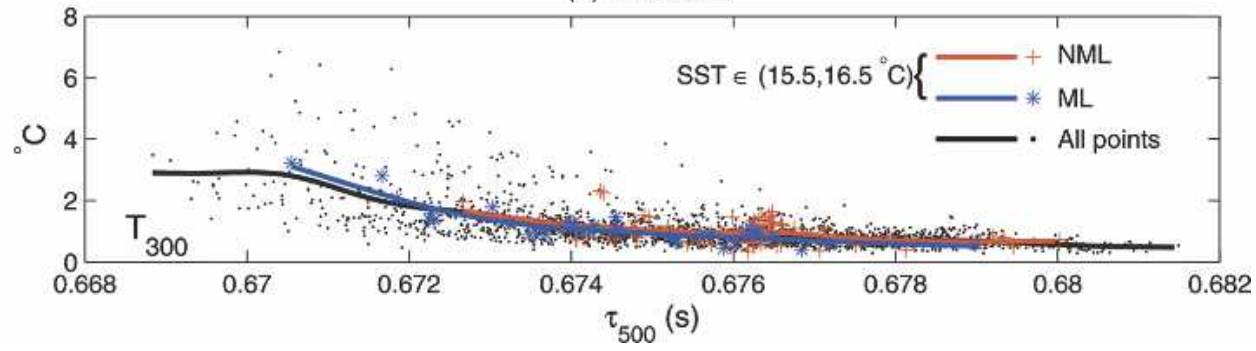

FIG. 3. Scatterplots of $T$ vs $\tau_{500}$ at (a) 100, (b) 200, and (c) $300 \mathrm{db}$. The superimposed black lines are the smoothed standard GEM curves fitted to all the 2248 historical hydrocasts (black dots). The superimposed red and blue lines are MI-GEM curves fitted to NML and ML hydrocasts (red crosses and blue asterisks) with SST between $15.5^{\circ}$ and $16.5^{\circ} \mathrm{C}$.

agrees with the monthly distribution of the number of NML and ML profiles. We choose 10 May as the date to switch from the ML to the NML MI-GEM fields. Although the ratio of NML to ML profiles is about 50:50 in September, the date to switch back from the NML to ML MI-GEM fields is chosen as 10 October, because within the UB most of the ML profiles are in this month. In addition, we included a 30-day transitional period that is centered on each of the two switching dates, during which time weighting functions for the two MI-GEM fields linearly increased and decreased (Fig. 5c), in order to produce smoothly varying profiles around the switching dates.

The OI-mapped SST data from the TRMM satellite, which are the fields we used as input to the MI-GEM, are compared with the SST data from the in situ CTD casts that are collected by KORDI and KODC. The TRMM SST data are interpolated to coincide with each CTD location. Figure 6 exhibits a scatterplot of CTD SST versus TRMM SST and a least squares fitted line that is superimposed on the plot. The two SST measurements are highly correlated with $\pm 0.957\left({ }^{\circ} \mathrm{C}\right)$ error bounds (gray-shaded zone) that contain the central
$50 \%$ of the points. Figure 6 demonstrates that the TRMM SST data are biased slightly high when the SST is lower than $\sim 15^{\circ} \mathrm{C}$. Therefore, we corrected the TRMM SST data using the linear relationship as (TRMM SST - 1.291)/0.947.

\section{b. Percent variance captured by MI-GEM fields}

The percent variance that is captured by the MIGEM fields for $T$ and $\delta$ as a function of pressure is calculated for each of the two variables by $1-\sigma_{\text {res }}^{2}(p) /$ $\sigma_{\text {tot }}^{2}(p)$, where $\sigma_{\text {res }}^{2}(p)$ is the variance of residuals of the 2248 CTDs from the MI-GEM fields, and $\sigma_{\text {tot }}^{2}$ is the total variance among the 2248 CTDs (Watts et al. 2001). Figure 7 compares the percent variance that is captured by the residual GEM and MI-GEM fields. Averaged through the depth range of $0-200 \mathrm{db}$, more than $92 \%$ and $88 \%$ of the variances in the observed $T$ and $\delta$ fields are captured by the MI-GEM. These percent variances are $3 \%$ and $4 \%$ higher, reducing by about one-third the unexplained error variance, than those of the residual GEM. Averaged through the depth range of 200-400 db, MI-GEM captures $73 \%$ and $67 \%$ of the variances for the two variables, which is an 


\section{(a) NML}

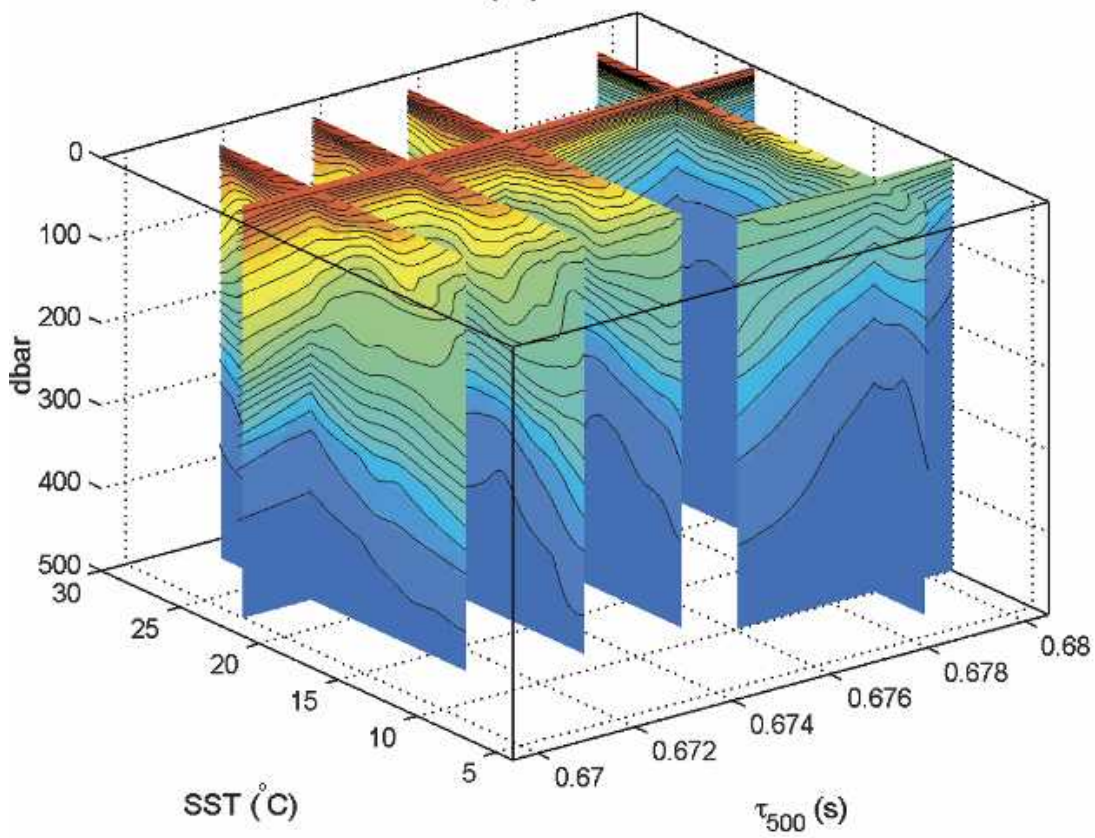

(b) ML

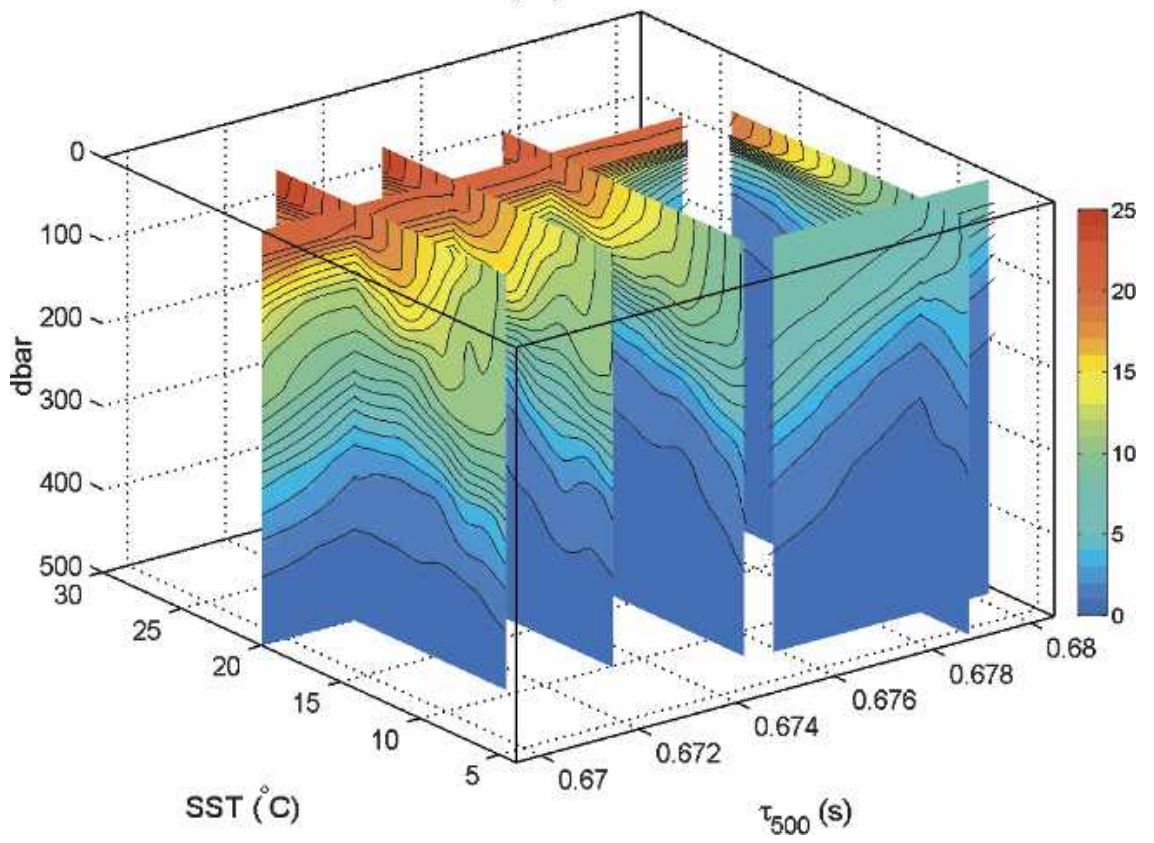

FIG. 4. MI-GEM $T$ fields for (a) non-mixed-layer and (b) mixed-layer groups. The color bar is temperature $\left({ }^{\circ} \mathrm{C}\right)$.

improvement over the residual GEM, which captures $68 \%$ and $60 \%$, respectively.

\section{Comparisons with observations}

The $441 T$ profiles from the in situ CTD casts (Fig. 1) that are collected by KORDI (305 casts) and KODC
(136 casts) are compared against profiles that are estimated from both the residual GEM and the MI-GEM. This is an independent comparison because those CTD casts were not used to construct the residual GEM and MI-GEM fields. The daily OI maps of $\tau_{500}$ and SST, described in section 2, were combined to estimate $T$ 
(a) Magnitude of basin average wind stress in the UB

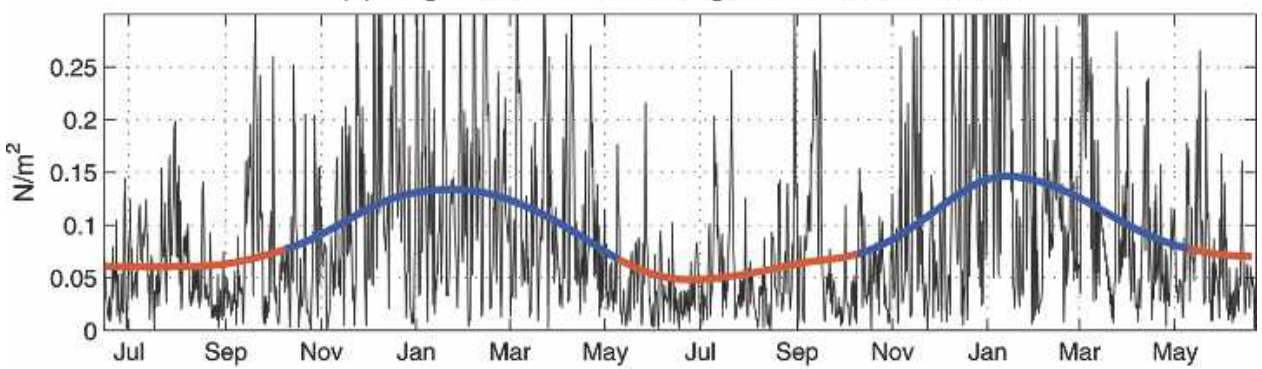

(b) Monthly distribution of historical hydrocasts

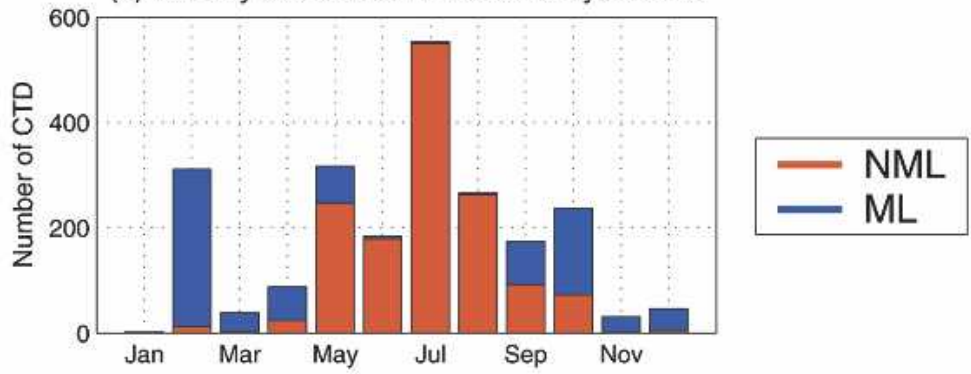

(c) Weighting Function

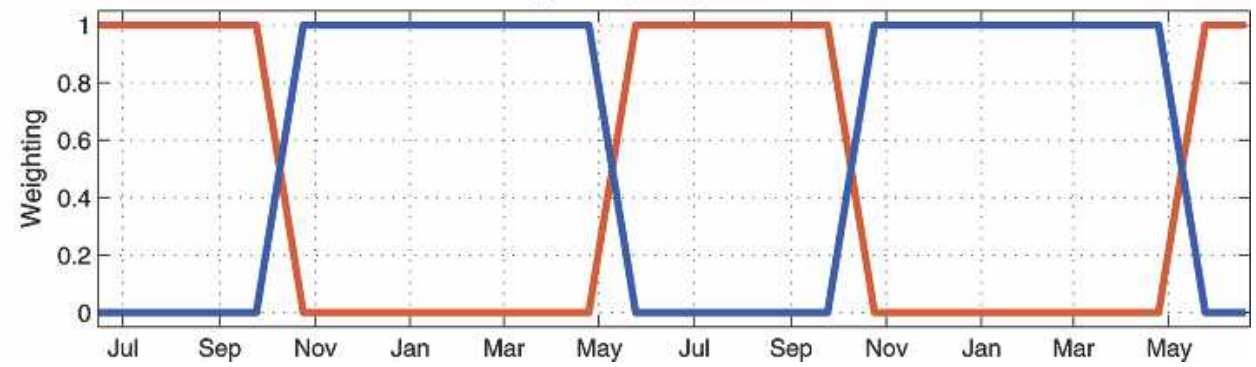

FIG. 5. (a) Magnitude of basin-averaged wind stress in the UB. The 90-day low-pass-filtered results are superimposed with red and blue curves, representing NML and ML periods, respectively. (b) Monthly distribution of NML (red) and ML (blue) groups from historical hydrocasts. (c) Weighting function between NML (red) and ML (blue) groups for MI-GEM application during the $2 \mathrm{yr}$ of observations.

profiles on a $0.125^{\circ} \times 0.125^{\circ}$ grid using the residual GEM and the MI-GEM lookup tables (OI-mapped SST errors are simulated in the appendix). These two sets of $T$ profiles were then interpolated horizontally to coincide with each CTD location. The difference between the estimated and actual $T$ was calculated at each 10-db level for all 441 CTD casts, and the rms residual is plotted in Fig. 8. The comparison reveals that PIESderived $T$ estimates using the MI-GEM have lower rms differences from the actual $T$ profiles, particularly in the 30-90- and 150-250-db-depth ranges.

The statistical improvement in Fig. 8 is small, because many of the 441 CTD profiles are well estimated by either technique. However, the improvement that is realized by the MI-GEM is significant at $20 \%-30 \%$ of the CTD stations. More importantly, it represents a qualitative improvement by eliminating false $T$ inversions and thermocline structures, as illustrated in the next two figures.

Figure 9 compares vertical $T$ sections from CTD casts along KODC lines 106 and 104, with coinciding (in space and time) PIES-derived $T$ sections calculated using the residual GEM and MI-GEM. The KODC observational surveys were conducted bimonthly and, hence, we have 12 comparisons of the $T$ sections at each KODC line. Figure 9 shows six examples of $T$ sections at two KODC lines, which exhibit the improvements of the MI-GEM estimates over the residual GEM.

The North Korea Cold Current and the East Korea Warm Current usually meet and form the subpolar front around $38^{\circ}-40^{\circ} \mathrm{N}$. KODC line $106\left(37.895^{\circ} \mathrm{N}\right)$ is near the southern boundary of the subpolar front and has been reported to cross the path of the ESIW (Kim and Kim 1983). The $T$ sections illustrate that the re- 


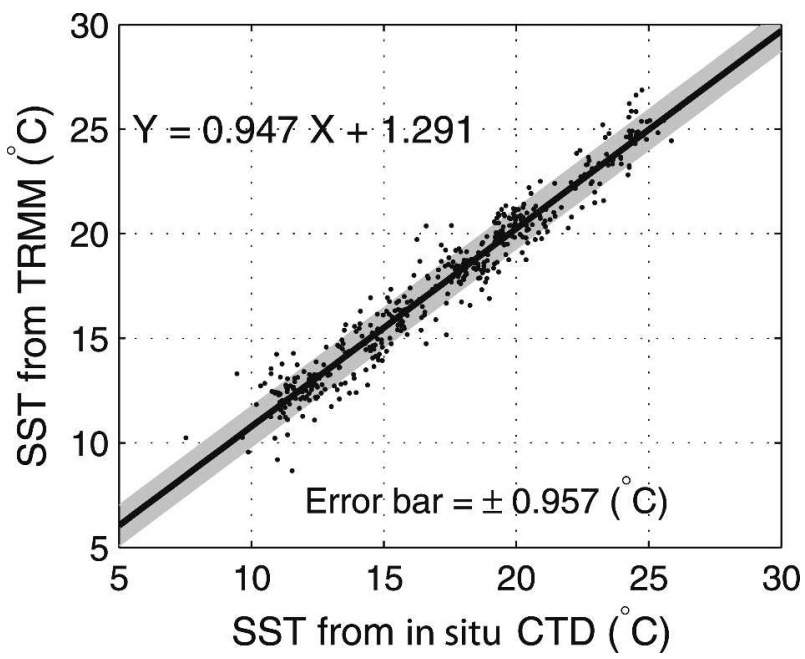

FIG. 6. Scatterplot of SST from in situ CTD vs SST from the TRMM satellite. The slope of a line fitted by least squares is 0.947 (thick line) and the gray-shaded zone indicates error bounds that contain at least $50 \%$ of the predictions.

sidual GEM underestimates the depth of the $1^{\circ} \mathrm{C}$ water surface $\left(Z_{1}\right)$, the lower boundary of the ESIW, at line 106, except during February 2000 (Fig. 9, left panels). In the August 1999 residual GEM $T$ section, the water layer between $1^{\circ}$ and $2^{\circ} \mathrm{C}\left(Z_{1,2}\right)$ is unrealistically thin around $129.5^{\circ}$ E. During August and October 2000, the $Z_{1,2}$ layer is too narrow along the whole transect. The April and June 2001 residual GEM $T$ sections have this same problem at the outer boundaries of the Ulleung warm eddy (UWE). All of these cases of narrow $Z_{1,2}$ occurred in regions where the thermocline is shallow. In contrast, the MI-GEM $T$ sections exhibit a more realistic $Z_{1,2}$ layer thickness in all cases and agree well with the CTD observations along line 106.

The UWE is usually centered near $37^{\circ} \mathrm{N}, 131^{\circ} \mathrm{E}$, and line 104 spans it along $37^{\circ} \mathrm{N}$. In the summertime, the residual GEM and MI-GEM $T$ sections both tend to agree with observations, as exemplified by the August 1999 section. In the wintertime, the surface layer of the UWE is homogenized by strong winds and the mixed layer deepens beyond $100 \mathrm{db}$, as exemplified in four CTD sections from December 1999, March and April 2000, and February 2001. In each case, the residual GEM $T$ sections contain unreasonable $T$ inversions between 30 and $100 \mathrm{db}$. The residual GEM $T$ section on line 106 for February 2000 contained the same fault in the upper $100 \mathrm{db}$. In contrast, the $T$ inversions are eliminated from the corresponding MI-GEM $T$ sections and the agreement with the CTD-derived ML depth and structure is qualitatively better. An additional improvement is illustrated in the June $2000 T$ section, in which a strongly developed lens-like eddy structure that was revealed by the CTD was replicated by the MIGEM, but not by the residual GEM.

Figure 10 compares the horizontal maps of $Z_{1}$ that are calculated from CTD surveys and PIES-derived maps, interpreted by each of the two GEM techniques for the same months as the KODC line 106 sections shown in Fig. 9. Along the northern edge, this provides a map view of the same faults with the residual GEM estimates that are "repaired" by the MI-GEM interpretation of the PIES data, as viewed in the vertical $T$ sections in Fig. 9. The August 1999, and August and October 2000 residual GEM $Z_{1}$ maps estimate unrealistically shallow $Z_{1}$ near $38^{\circ} \mathrm{N}$, which disagrees with the CTD $Z_{1}$ maps. The April and June 2001 residual GEM maps illustrate excessively steep changes of $Z_{1}$ at the eastern and western boundary of the UWE. The MIGEM $Z_{1}$ maps eliminate the shallow $Z_{1}$, and agree well with the CTD $Z_{1}$ maps for all of the compared cases.

In other portions of these maps (Fig. 10) two other types of error of the residual GEM estimates are found. In the February 2000 and April 2001 maps, the spatial extent of the UWE in the residual GEM maps is too large when compared with that in the CTD and MIGEM maps. This happens because the residual GEM overestimates $Z_{1}$ in the western and southern parts of the UWE. The residual GEM estimates also err significantly in the southwestern corner of the mapping domain, where they generate a feature with $Z_{1}$ that is deeper than $400 \mathrm{~m}$. This unrealistic feature does not occur in the CTD and MI-GEM $Z_{1}$ maps. Figure 11 demonstrates that this erroneous feature originates from a bias of the MODAS dataset, which is the reference climatology of the residual GEM. The MODAS vertical $T$ section along $130^{\circ} \mathrm{E}$ in March illustrates this problem south of $36^{\circ} \mathrm{N}$, where $Z_{1}$ dives unrealistically deep. This error produced the isolated deep cold water mass that is estimated by the residual GEM.

\section{Summary and discussion}

Our objective has been to interpret data from a PIES array that was deployed for $2 \mathrm{yr}$ in the Ulleung Basin of the southwestern JES. We wanted to improve upon estimates of $T$ and $\delta$ profiles that were obtained using the standard GEM technique, because variations in the shallow permanent thermocline that are caused by eddies are difficult to separate from the spatiotemporally varying annual signal. Mitchell et al. (2004) addressed this difficulty by developing a residual GEM technique. That technique demonstrated considerable success in mapping the 100-m $T$ field, which has served as an effective proxy for mapping currents and eddies in the JES. However, the residual GEM technique still pro- 
(a)

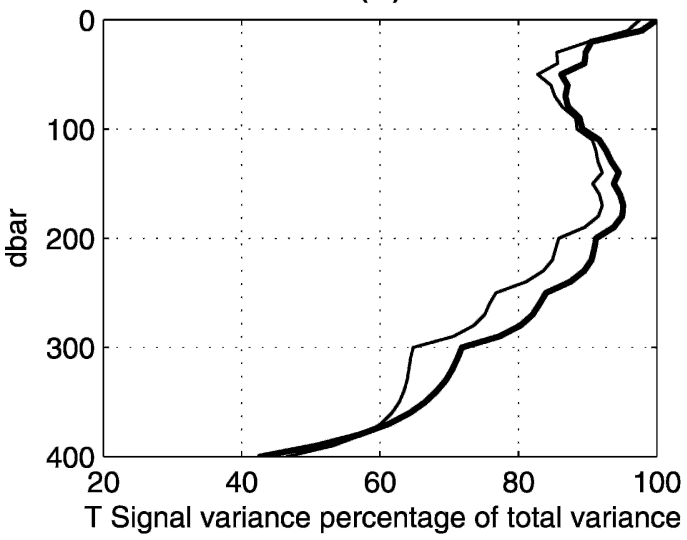

(b)

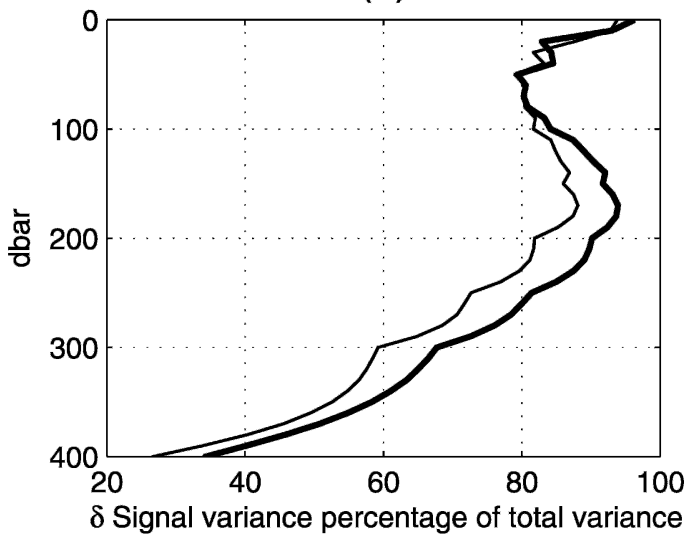

FIG. 7. Percent of (a) $T$ and (b) $\delta$ signals captured by the residual GEM (thin line) and MI-GEM (thick line).

duced several erroneous features, with the most problematic being significant $T$ inversions within the nearsurface layer in the wintertime. That technique also estimated an unreasonably thin intermediate water layer in regions where the thermocline was very shallow. Unrealistically thick intermediate water layers were also obtained in one region where the MODAS climatology profiles have bias errors as demonstrated by comparisons with concurrent in situ CTD observations.

From our subsequent studies, we now understand that because the residual GEM technique profiles of the $T$ and $\delta$ anomaly (relative to MODAS climatology) have subsurface extrema, when these are associated with a different residual $\tau$ ( $\tau$ anomaly) and added back

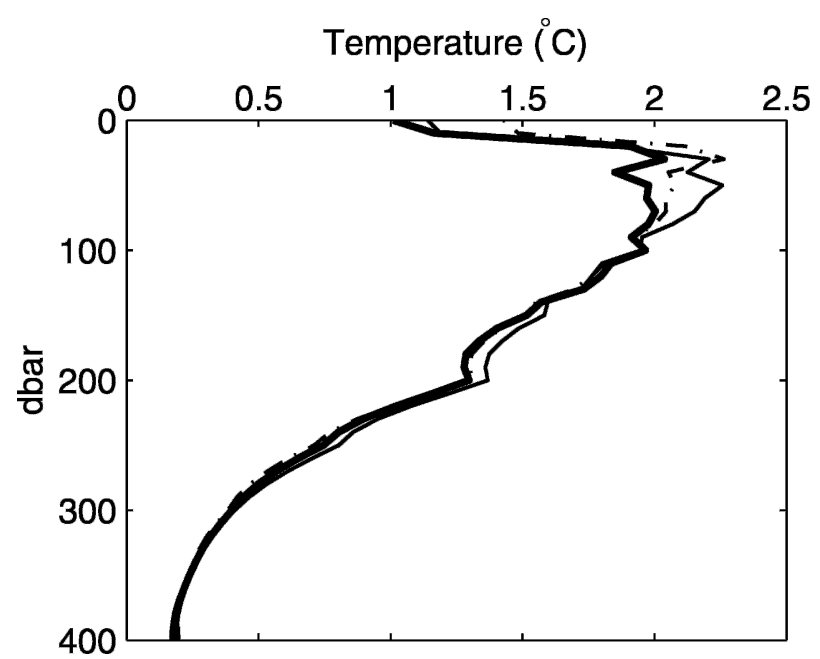

FIG. 8. The rms differences of $T$ between 441 CTD profiles and PIES-derived profiles estimated by the residual GEM (thin line) and the MI-GEM (thick line). The dashed-dotted line simulates added SST error, as explained in the appendix. to the smooth MODAS climatological profiles, the summed estimate can generate false inversions. The subsurface positive (negative) anomaly extremum that is estimated when a large negative (positive) residual $\tau$ occurs is typically associated with a strong, warm (cold) eddy. For example, during the wintertime, the UWE has a large negative $\tau$ anomaly. The residual GEM corrects the climatological $T$ profiles by adding a positive subsurface extremum, which cause an unreal subsurface $T$ inversion, as examplified in Fig. 9. Yet none of the original data profiles have subsurface $T$ inversions. So, we returned to seek a technique that developed GEM fields directly from the original data profiles rather than anomaly profiles. However, we also simultaneously addressed the shallow pycnocline problem (of separating the eddy signal from the annual signal) by including additional information that was available from SST and the temporal presence or absence of near-surface mixed layers in the historical hydrocasts. We selected the SST bins having $1^{\circ} \mathrm{C}$ intervals from $5^{\circ}$ to $30^{\circ} \mathrm{C}$, while Mitchell et al. (2004) classified the residual SST into only three bins.

We call this new technique an MI-GEM, parameterized by $\tau_{500}$, SST, ML information, and pressure to make multi-index lookup tables for $T$ and $\delta$ from historical hydrocasts. To parameterize the ML strength, the historical hydrocasts are regrouped into NML and ML groups. The appropriate dates for NML and ML fields are determined by the monthly distribution of the number of NML and ML profiles in the historical hydrocasts, which also correlated with the strength of the 90-day low-pass-filtered winds during our observation. The MI-GEM technique reduced by about one-third the unexplained error variance of the residual GEM. Although the MI-GEM technique includes the ML information, it is still only an estimate of the ML struc- 

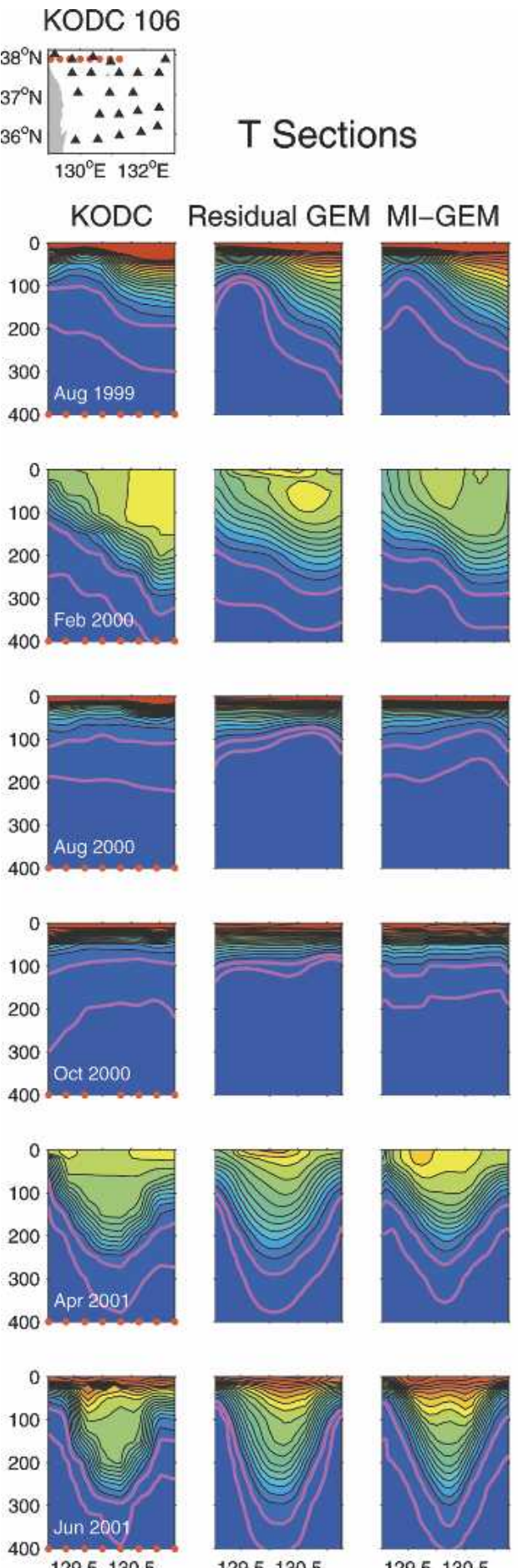
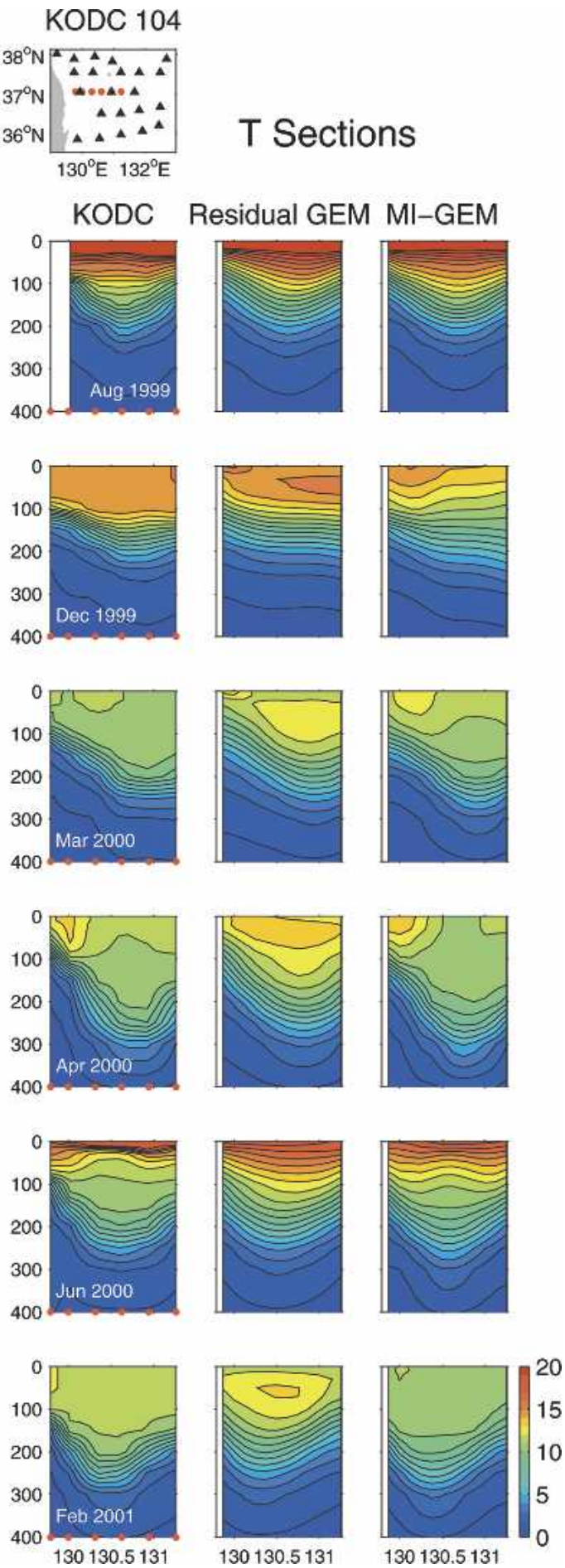

130130.5131

FIG. 9. Vertical $T$ sections along lines (left) 106 and (right) 104 from KODC bimonthly surveys of serial oceanographic stations (red dots) compared to those estimated by the residual GEM and the MI-GEM techniques. Magenta lines in line 106 sections indicate the $Z_{1,2}$ layer boundaries $\left(1^{\circ}, 2^{\circ} \mathrm{C}\right)$. The color bar is temperature $\left({ }^{\circ} \mathrm{C}\right)$, and the contour interval is $1^{\circ} \mathrm{C}$.

ture, and this remains the largest source of unexplained error variance.

The MI-GEM technique is applied to the $\tau$ measured by a PIES array in the UB to produce water column estimates of $T$ and $\delta$ from 0 to $500 \mathrm{db}$. At first sight, the comparison of $441 T$ profiles from in situ CTD casts against coinciding residual GEM and MI-GEM $T$ profiles reveals only small a quantitative improvement. 
KODC stations

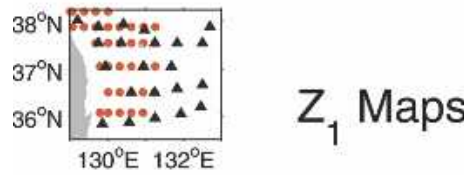

KODC Residual GEM MI-GEM
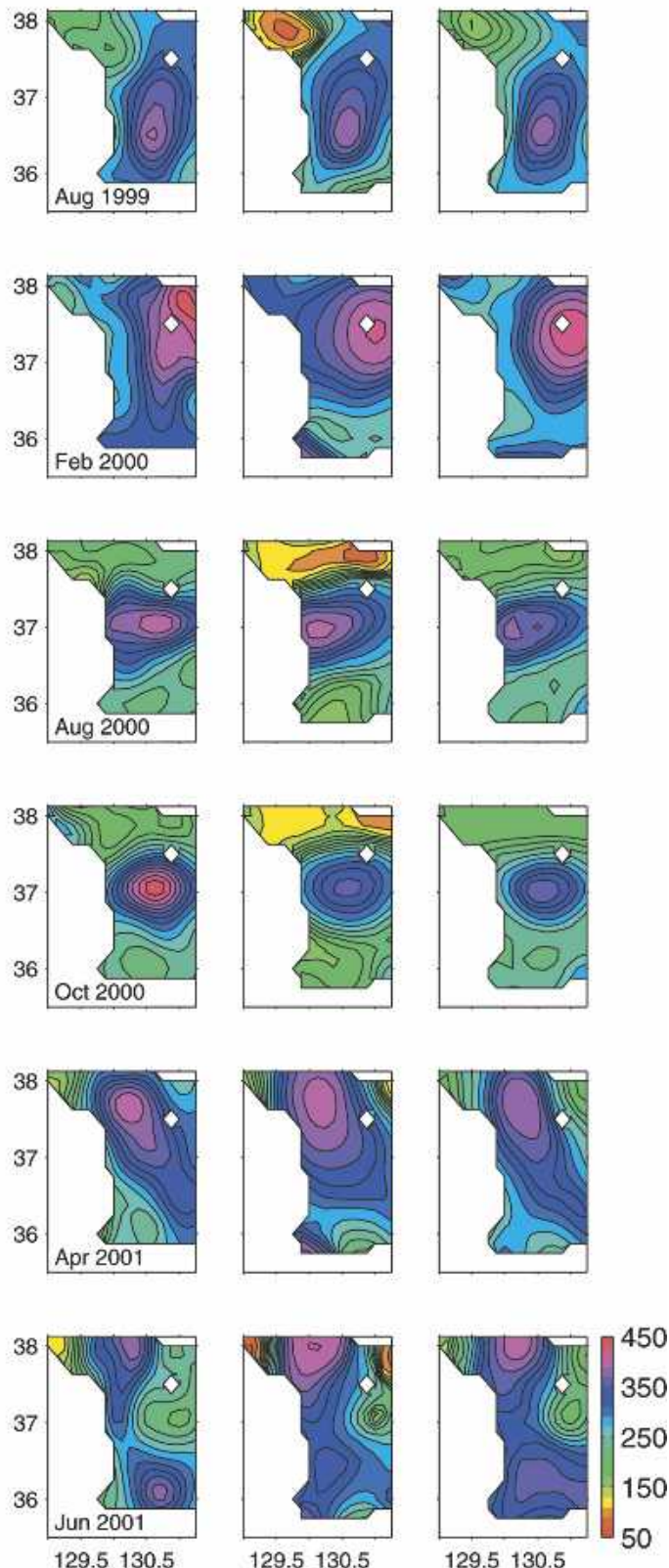

FIG. 10. Horizontal maps of $Z_{1}$ calculated from KODC bimonthly surveys (red dots) compared to those from the residual GEM and the MI-GEM estimates. The color bar is depth (m), and the contour interval is $20 \mathrm{~m}$.

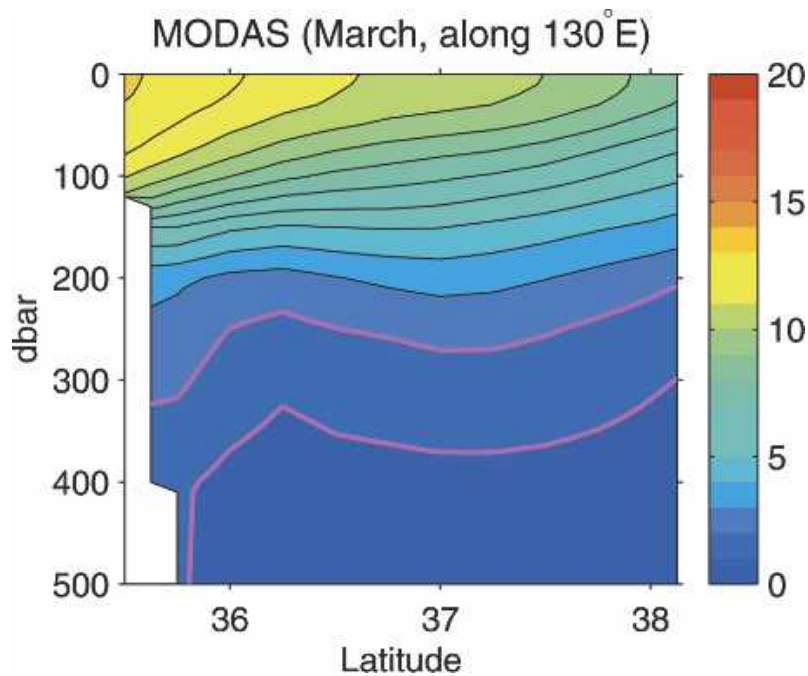

FIG. 11. A vertical $T$ section of MODAS along $130^{\circ} \mathrm{E}$ in Mar, illustrating a problem in the MODAS $T$ field south of $36^{\circ} \mathrm{N}$. Magenta lines indicate the $Z_{1,2}$ layer boundaries $\left(1^{\circ}, 2^{\circ} \mathrm{C}\right)$.

Moreover, the $T$ maps at a 100-m depth from the MIGEM estimates are almost identical to those from the residual GEM estimates (not shown in this paper). However, the MI-GEM technique eliminates unrealistic $T$ inversions and thin or thick intermediate water layers, which were misestimated by the residual GEM. These significant qualitative improvements are demonstrated by comparisons of vertical $T$ sections and horizontal $Z_{1}$ maps that are calculated from independent KODC bimonthly in situ CTD surveys. The small reduction in scatter around the MI-GEM fields, as shown in Fig. 5, results in smaller error bars on the estimated vertical $T$ and $\delta$ profiles from $\tau$ and, hence, the MIGEM technique almost always produced improved upper water column profiles of $T$ and $\delta$ compared to the residual GEM technique. The qualitative improvement of the intermediate water layer estimates provides us an opportunity to investigate the ESIW dynamics using daily maps of the ESIW circulation pattern in future work.

For the above reasons the MI-GEM is clearly preferred over the residual GEM for Ulleung Basin studies. Three points address the naturally arising question as to whether the MI-GEM technique should outperform other techniques in all ocean regions. 1) The obvious point is that multiple-index proxies should always reduce the unexplained variance compared to the standard two-index GEM, provided that one selects additional indices that independently account for a portion of the observed variability. 2) It remains possible that a residual GEM may provide better estimates of vertical profiles in a location where a good climatology is avail- 
able and the climatological annual signal is large compared to the mesoscale eddy signal. Residual GEM fields might also be improved by grouping stations as we did here. Nevertheless, the residual GEM technique should always be approached cautiously, because the anomaly fields that it generates may contain subsurface extrema, which are intrinsically subject to the problem of estimating unrealistic subsurface temperature and density features. 3) The lookup tables that are generated by the MI-GEM technique (and the standard GEM) are composed directly from real hydrographic profiles. Every input profile is stably stratified and contributes a point to every estimated output depth. Hence, the MI-GEM smoothed lookup tables should be relatively immune to containing unreal features. Moreover, the MI-GEM fields may be examined directly, rather than as anomaly fields; and if some unrealistic feature were generated it would come to a user's attention readily.

In regions where the seasonal variations are sufficiently decoupled vertically from the large-scale pycnocline variations, the standard GEM (Meinen and Watts 2000 ) and/or the improved standard GEM (Watts et al. 2001) will probably satisfactorily capture a high percentage of the variance of the temperature and specific volume anomaly profiles. In all regions of the ocean, it seems likely that the GEM lookup tables can be improved by using additional proxy indices to reduce the residual variance. This paper has demonstrated the particular advantages of combining $\tau$ data with satellite SST and ML information in an MI-GEM.

Acknowledgments. We thank K.-I. Chang for providing high-resolution CTD profiles. We would also like to thank Mark Wimbush and two anonymous reviewers whose useful comments helped us to improve the quality of this paper. This work was supported by the Office of Naval Research "Japan/East Sea DRI," including two basic research programs-the Japan/East Sea initiative under Grant N000149810246, and the Naval Research Laboratory's "Linkages of Asian Marginal Seas" under Program Element 0601153N.

\section{APPENDIX}

\section{Simulated Introduction of SST Errors}

To evaluate the impact of errors in the OI-mapped TRMM SST data, we conducted a simulation of estimating $T$ profiles using the MI-GEM technique after adding a $1^{\circ} \mathrm{C}$ error to all of the SST data. The estimated
$T$ profiles were interpolated to coincide with each CTD location, and then the rms residual between estimated and actual $T$ was included in Fig. 8 (dashed-dotted line). This simulated additional SST error increased the rms residual in the upper $100 \mathrm{~m}$. However, even if the SST data had a $1^{\circ} \mathrm{C}$ error added, the $T$ profiles that were estimated from the MI-GEM were still better than those estimated from residual GEM without the SST error.

\section{REFERENCES}

Book, J. W., M. Wimbush, S. Imawaki, H. Ichikawa, H. Uchida, and H. Kinoshita, 2002: Kuroshio temporal and spatial variations south of Japan determined from inverted echo sounder measurements. J. Geophys. Res., 107, 3121, doi:10.1029/ 2001JC000795.

Kim, C. H., and K. Kim, 1983: Characteristics and origin of the cold water mass along the east coast of Korea (in Korean with English abstract). J. Oceanol. Soc. Korea, 18, 73-83.

- H.-J. Lie, and K.-S. Kim, 1991: On the intermediate water in the southeastern East Sea (Sea of Japan). Oceanography of Asian Marginal Seas, K. Takano, Ed., Elsevier Science, 129141.

Kim, K., and J. Y. Chung, 1984: On the salinity-minimum and dissolved oxygen-maximum layer in the East Sea (Sea of Japan). Ocean Hydrodynamics of the Japan and East China Seas, T. Ichiye, Ed., Elsevier Science, 55-65.

Meinen, C. S., and D. R. Watts, 1997: Further evidence that the sound speed algorithm of Del Grosso is more accurate than that of Chen and Millero. J. Acoust. Soc. Amer., 102, 2058 2062.

—_, and ——, 1998: Calibrating inverted echo sounders equipped with pressure sensors. J. Atmos. Oceanic Technol., 15, 1339-1345.

— transect across the North Atlantic Current near $42^{\circ} \mathrm{N}$ : Time series and mean. J. Geophys. Res., 105, 21 869-21 891.

Mitchell, D. A., M. Wimbush, D. R. Watts, and W. J. Teague, 2004: The residual GEM technique and its application to the southwestern Japan/East Sea. J. Atmos. Oceanic Technol., 21, 1895-1909.

— , and Coauthors, 2005: Upper circulation patterns in the southwestern Japan/East Sea. Deep-Sea Res., in press.

Shin, C. W., S. K. Byun, C. S. Kim, and Y. H. Seung, 1998: Southward intrusion of the East Sea Intermediate Water into the Ulleung Basin: Observations in 1992 and 1993. J. Korean Soc. Oceanogr., 33, 146-156.

Sun, C., and D. R. Watts, 2001: A circumpolar gravest empirical mode for the southern ocean hydrography. J. Geophys. Res., 106, 2833-2855.

Tracey, K. T., S. D. Howden, and D. R. Watts, 1997: IES calibration and mapping procedures. J. Atmos. Oceanic Technol., 14, 1483-1493.

Watts, D. R., C. Sun, and S. Rintoul, 2001: A two-dimensional gravest empirical mode determined from hydrographic observations in the Subantarctic Front. J. Phys. Oceanogr., 31, 2186-2209. 